\title{
A AUSÊNCIA MASCULINA NA ATENÇÃo PRIMÁRIA À SAÚDE: UMA ANÁLISE DA TEORIA DA AÇÃo PLANEJADA
}

Jéssica Bazilio Chaves

Universidade Federal de Alagoas

\author{
Sheyla Christine Santos Fernandes \\ Universidade Federal de Alagoas
}

Daniela Santos Bezerra

Universidade Federal de Alagoas

\begin{abstract}
Resumo
Este estudo apresenta, a partir da Teoria da Ação Planejada, uma análise sobre a ausência masculina na Atenção Primária à Saúde. Objetivou-se identificar as crenças positivas e negativas e definir as crenças modais salientes relativas ao comportamento de "procurar a Unidade Básica de Saúde" de homens de um distrito sanitário de Maceió $(\mathrm{AL})$. Participaram do estudo 25 homens, entre 25 e 59 anos, cadastrados em uma Unidade Básica de Saúde (UBS) de um distrito sanitário de Maceió. Utilizou-se entrevista semiestruturada e adotou-se análise de conteúdo de Bardin. Os homens apresentaram crenças comportamentais que tanto favorecem como dificultam o comportamento. As crenças normativas demonstraram maior influência das mulheres para a adoção do comportamento. As crenças de controle foram representadas pelos participantes como negativas, mesmo que algumas delas tenham facilitado a realização do comportamento. Isto comprova que a procura masculina à UBS está relacionada às crenças que envolvem o seu universo cognitivo.
\end{abstract}

Palavras-chave: Saúde do homem; comportamento; atenção primária à saúde; teoria da ação planejada.

\section{THE MEN'S ABSENCE IN PRIMARY HEALTH CARE: AN ANALYSIS OF THE THEORY OF PLANNED ACTION}

\begin{abstract}
This study presents, from the Theory of Planned Action, an analysis of male absence in Primary Health Care. The objective was to identify positive and negative beliefs and to define the salient modal beliefs related to the behavior of "seeking the Basic Health Unit "Of men from a sanitary district of Maceió (AL). A total of 25 men, aged 25-59 years, enrolled in one Basic Health Unit (BHU) of a health district of Maceió participated in the study. A semi-structured interview was used and Bardin content analysis was adopted. Men presented behavioral beliefs that both favor and hinder behavior. Normative beliefs have shown greater influence of women for behavioral adoption. The control beliefs were represented by the participants as negative, even though some of them facilitated the performance of the behavior. This proves that male demand for $\mathrm{BHU}$ is related to the beliefs that surround their cognitive universe. Keywords: Men's health; behavior; primary health care; theory of planned action.
\end{abstract}




\title{
AUSENCIA DE LOS HOMBRES EN ATENCIÓN PRIMARIA DE SALUD: UN ANÁLISIS DE LA THEORY OF PLANNED ACTION
}

\begin{abstract}
Resumen
Este estudio presenta, a partir de la Teoría de Acción Planificada, un análisis de la ausencia masculina en Atención Primaria de Salud. El objetivo fue identificar las creencias positivas y negativas y definir las creencias modales sobresalientes relacionadas con el comportamiento de "buscar la Unidad Básica de Salud" de hombres de un distrito sanitario de Maceió (AL). Un total de 25 hombres, de 25 a 59 años, matriculados en una Unidad Básica de Salud (UBS) de un distrito de salud de Maceió participaron en el estudio. Se utilizó entrevista semiestructurada y se adoptó el análisis de contenido de Bardin. Los hombres presentaban creencias conductuales que favorecían y obstaculizaban el comportamiento. Las creencias normativas han demostrado una mayor influencia de las mujeres en la adopción del comportamiento. Las creencias de control fueron representadas por los participantes como negativas, aunque algunas de ellas facilitaron el desempeño del comportamiento.

Palabras-clave: Salud de los hombres; comportamiento; atención primaria de salud; teoría de la acción planeada.
\end{abstract}

\section{INTRODUÇÃO}

As estatísticas epidemiológicas vêm apresentando uma discrepância na expectativa de vida de homens e mulheres e esse dado tem fortalecido o estudo sobre a saúde masculina no Brasil (Mendonça \& Andrade, 2010). De acordo com o Instituto Brasileiro de Geografia e Estatística (IBGE), a taxa de mortalidade se apresenta diferenciada entre os sexos, sendo que a masculina é sempre superior à feminina. Em 2015 a expectativa de sobrevida dos homens era de 71,9 anos, enquanto que para as mulheres correspondia a 79,1 anos. O levantamento apontou, ainda, que Alagoas tem a menor esperança de vida para os homens $(66,5$ anos $)$. O estado de Alagoas também apresenta a maior diferença na expectativa de vida entre homens e mulheres, com 9,5 anos a mais a favor das mulheres (IBGE, 2016). Resultados desse tipo impulsionam a literatura a adotar a perspectiva relacional de gênero, a fim de explicar os processos de saúde e doença que normalmente permeiam o grupo masculino (Alves, Silva, Ernesto, Lima, \& Souza, 2011; Couto et al., 2010; Nascimento \& Lemes, 2014; Storino, Souza, \& Silva, 2013).

Atribui-se à cultura masculina, com suas formas de vida e de socialização, como a principal causadora dos problemas relacionados à saúde dessa população (Gomes et al., 2011; Santos, 2010). Acredita-se na persistência de um modelo de masculinidade hegemônico, que trata a relação entre os gêneros como desigual e de poder, estimulando os homens a cuidarem menos de si e a se exporem mais aos riscos, visto que the são atribuídas características como "forte", "viril" e "invulnerável" (Gomes et al., 2011; Schraiber et al., 2010). Desse modo, o fato de se considerarem como invulneráveis aos agravos torna difícil a entrada dos homens nos Serviços de Atenção Primária à Saúde (Albano, Basílio, \& Neves, 2010).

Sabo (2002) pontua que os riscos aos quais os homens se expõem estão normalmente associados à violência, consumo excessivo de álcool e outras 
drogas. Além desses, também se destacam o envolvimento com esportes e trabalhos que exigem mais do corpo, mais arriscados, e questões que envolvem a virilidade e sexualidade. Quanto aos cuidados com a saúde, evidências empíricas (Alves et al., 2011; Gomes et al., 2011) atestam que homens reconhecem e expressam menos as suas necessidades, acreditam que precisam menos de assistência e buscam menos os serviços de saúde quando comparados às mulheres. A justificativa encontrada na perspectiva de gênero demonstra que as crenças e valores existentes no universo masculino remetem ao cuidado um papel tipicamente feminino. Em relação às necessidades de saúde, estas representam fraqueza e fragilidade (atributos femininos) e os serviços de saúde seriam locais específicos para mulheres, devido à sua acessibilidade, estrutura e funcionamento (Alves et al., 2011; Gomes et al., 2011).

De acordo com o Ministério da Saúde (2009), a não procura pelos serviços de Atenção Primária à Saúde (APS) faz com que os homens fiquem privados da proteção necessária à preservação de sua saúde, continuando a fazer uso de procedimentos desnecessários. Muitos agravos e custos ao sistema de saúde pública poderiam ser evitados caso os homens realizassem com regularidade as medidas de prevenção primária. Desse modo, tem sido encontrada uma maior prevalência dos homens em serviços emergenciais, como os prontos-socorros e farmácias. Nestes espaços, as demandas são respondidas mais objetivamente, os problemas se tornam mais fáceis de serem expostos e, na maioria das vezes, não é necessário enfrentar filas ou marcar consultas (Gomes et al., 2011; Pinheiro, Viacava, Travassos, \& Brito, 2002).

Outras barreiras associadas a essa invisibilidade masculina estão ligadas ao próprio serviço, no caso, as Unidades Básicas de Saúde (UBS's), devido aos seus horários de funcionamento, considerados pela maioria como não condizentes com a carga horária de trabalho. Aliado a isso, os homens visualizam estes locais e os seus programas como sendo destinados às crianças, mulheres e idosos. Além disso, receiam 0 fato das equipes serem majoritariamente compostas por mulheres (Alves et al., 2011; Gomes et al., 2011). A dificuldade na marcação de consultas médicas e exames, assim como a escassez de profissionais e médicos especialistas, também são fatores que levam a julgamentos negativos por parte dos homens em relação aos serviços de Atenção Primária à Saúde (Azevedo, 2007; Brito, Santos, \& Maciel, 2010).

Diante desse cenário, o Ministério da Saúde criou em 2009 a Política Nacional de Atenção Integral à Saúde do Homem (PNAISH), com objetivo de promover a melhoria das condições de saúde da população masculina do Brasil. Além disso, a PNAISH visa contribuir para a redução da morbidade e mortalidade dessa população, através do enfrentamento racional dos fatores de risco e mediante a facilitação do acesso às ações e aos serviços de assistência integral à saúde (Alves et al., 2011; Ministério da Saúde, 2009; Moura, Santos, Neves, Gomes, \& Schawrz, 2014). Essa política tem como foco principal dar assistência 
aos homens na faixa etária dos 25 aos 59 anos, uma vez que se trata de uma parcela preponderante da força produtiva, com significativo papel sociocultural e político, além de nunca ter sido incluída em nenhuma outra política de saúde (Alves et al., 2011; Ministério da Saúde, 2009).

Portanto, como afirmam Alves et al. (2011), é necessário conhecer os aspectos psicossociais que influenciam os homens na hora de procurarem os serviços da Atenção Primária à Saúde. Isso pode contribuir com a proposição de mudanças institucionais, capazes de atrair esse público, interferindo nas construções culturais ligadas aos cuidados com a saúde e as percepções dos homens em relação a esses cuidados.

A partir da perspectiva de que os homens não procuram as UBS's, e que isso pode estar atrelado às crenças que permeiam o universo desse público, optou-se por utilizar o olhar da Teoria da Ação Planejada (TAP) de Fishbein e Ajzen (1975). A Teoria da Ação Planejada foi desenvolvida como extensão da Teoria da Ação Racional (TAR), criada em 1967 por Martin Fishbein e revisada e aprofundada por Fishbein e Ajzen na década de 1970 (Fishbein \& Ajzen, 1975). A TAR considera que as pessoas, como seres racionais, normalmente agem de acordo com as suas intenções. Para isso, utilizam as informações disponíveis e avaliam as implicações de seus comportamentos, a fim de decidirem por sua realização. Portanto, o seu objetivo é compreender e predizer um determinado comportamento (Fishbein \& Ajzen, 1975).

Sendo assim, de acordo com a TAR, a intenção de realizar um comportamento é resultante de uma avaliação pessoal positiva das possíveis consequências desse comportamento e da percepção de que ele será socialmente aprovado. A intenção, nessa teoria, é denominada Intenção Comportamental e está sob influência de dois determinantes básicos: um pessoal e outro social. 0 determinante pessoal diz respeito à Atitude em relação ao comportamento, enquanto que o social se relaciona com a percepção pelo sujeito das pressões sofridas na realização ou não dos comportamentos, sendo esse fator denominado Norma Subjetiva (Ajzen \& Fishbein, 1980; D'amorim, 2000; Fishbein \& Ajzen, 1975).

As crenças formam a base da TAR e são entendidas como subjacentes às Atitudes e às Normas Subjetivas, o que, em última instância, as colocam como determinantes de Intenções e Comportamentos (Moutinho \& Roazzi, 2010). Para Fishbein e Ajzen (1975), as crenças referem-se às percepções dos sujeitos em relação a algum aspecto existente no seu mundo. As crenças formam a compreensão que os sujeitos elaboram a respeito de si e do meio que os cercam. Representam a informação por eles acumuladas ao longo de suas experiências de vida em relação ao comportamento específico, seja essa informação correta ou incorreta, seja pertinente ou irrelevante. Em síntese, as crenças representam a informação que o sujeito tem sobre um determinado objeto, relacionando a ele algum atributo. Consideram-se como objetos: pessoas, grupos, instituições, 
comportamentos etc. Enquanto que os atributos podem ser: uma qualidade, consequência, característica ou evento. Por exemplo, no estudo de Gonçalves e Dias (1999) as mulheres entrevistadas atribuíram ao comportamento (objeto) "realizar o autoexame da mama" o atributo "encontrar nódulos e caroços".

No entanto, o modelo da TAR apresentou limitações, uma vez que os comportamentos que não estavam sob o controle volitivo ou motivacional das pessoas eram desconsiderados, podendo o comportamento ser influenciado por outros fatores, como o hábito (por exemplo, sujeitos que não conseguiram parar de fumar na primeira tentativa). A dificuldade de se conseguir controlar o próprio comportamento quando outras variáveis podem ter um papel importante nessa determinação pode impedir a ação de acontecer. Dessa forma, Ajzen buscou a ampliação e expansão da Teoria da Ação Racional através da Teoria da Ação Planejada (Ajzen, 1991; Moutinho \& Roazzi, 2010).

O novo modelo da teoria, para além do que defendia a TAR, acrescenta outro elemento de previsão, a Percepção de Controle. Esta se caracteriza como a percepção acerca da presença de fatores que podem facilitar ou impedir a performance do comportamento. Pressupõe-se, portanto, que uma maior percepção de controle do comportamento corresponde a uma maior probabilidade de que o seu desempenho tenha sucesso (Ajzen, 1991).

Portanto, a Atitude é considerada como uma predisposição para responder de alguma forma preferencial, ou seja, o quanto o sujeito é favorável ou não ao desempenho do comportamento avaliado. A Norma subjetiva corresponde à percepção do indivíduo quanto à pressão social exercida sobre o mesmo para que realize ou não o comportamento em questão. A Percepção de Controle é definida como sendo as crenças do indivíduo acerca do grau de facilidade/dificuldade em executar uma determinada ação, isto é, à percepção de poder executar um comportamento desejado. Desta forma, os construtos Atitudes, Norma Subjetiva e Percepção de Controle são determinados respectivamente pelas Crenças Comportamentais, Crenças Normativas e Crenças de Controle e pelo poder de cada uma em influenciar o indivíduo (Ajzen, 1991).

Resumindo, em suas áreas específicas, as Crenças Comportamentais produzem uma Atitude favorável ou desfavorável em relação ao comportamento. As Crenças Normativas são expressões da pressão social percebida ou das Normas Subjetivas e as Crenças de Controle são o resultado do controle percebido sobre o comportamento (Moutinho \& Roazzi, 2010). De forma combinada, as três levam à formação das Intenções Comportamentais. As Intenções sendo fortes e bem estabelecidas levam a expectativas realistas (Ajzen, 1991).

A TAP é um modelo bastante utilizado em pesquisas não só na área da Psicologia, como também em diversas outras áreas que se preocupam com adoção de comportamentos preventivos em saúde. Em razão da possibilidade de conhecimento das intenções de um dado comportamento, em uma determinada 
população, permite aos programas e políticas de saúde a utilização de estratégias mais eficazes e resolutivas (Moutinho \& Roazzi, 2010).

Diversos estudos que utilizam a TAP como referencial teórico vêm sendo realizados na literatura nacional e internacional. Por exemplo, Courneya e Friedenreich (1999) realizaram um estudo sobre adoção do exercício físico em pacientes com câncer. De forma semelhante, outros estudos buscaram investigar a adoção da prática de atividades físicas e de lazer (Armitage, 2005; Courneya, 1995; Maciel \& Veiga, 2012). A adoção de comportamentos preventivos também é bastante discutida a partir da utilização da TAP. Por exemplo, o estudo de Conner, Warren, Close e Sparks (1999) a respeito da prevenção ao consumo de álcool. Seguindo essa mesma linha de investigação, identifica-se o estudo de Harakeh, Scholte, Vermulst, Vries e Engels (2004) sobre prevenção ao fumo e o estudo de D'amorim, Freitas e Sá (1992) sobre prevenção ao câncer de mama. Outros estudos também apresentaram como interesse ampliar a discussão sobre a adoção de comportamentos relacionados às questões de saúde. Por exemplo, o uso de preservativo entre adolescentes (Bryan, Fisher, \& Fisher, 2002; Matos, Veiga, \& Reis, 2009) e a adoção de alimentação saudável (Conner, Norman \& Bell, 2002).

Considerando a importância de aprofundar o debate acerca da ausência masculina na APS a partir de uma perspectiva de crenças, o presente estudo teve como objetivos: identificar as crenças positivas e negativas e definir "as crenças modais salientes" relativas ao comportamento de "procurar a Unidade Básica de Saúde" de homens de um distrito sanitário de saúde de Maceió $(A L)$. O presente estudo pretende contribuir com dados que proporcionem aos gestores de saúde locais a busca de alternativas que promovam uma maior adesão masculina aos serviços de Atenção Primária à Saúde.

\section{MÉTODO}

\section{Caracterização da Pesquisa}

Trata-se de um estudo exploratório e descritivo de abordagem qualitativa, a partir de uma amostragem não probabilística, considerando um dos oito distritos sanitários de Maceió ( $A L)$.

\section{Participantes}

A amostra foi composta por 25 participantes cadastrados em uma das cinco Unidades Básicas de Saúde de um distrito sanitário de Maceió, caracterizando uma amostra não probabilística por conveniência, sendo todos homens entre 25 e 59 anos de idade (faixa etária do público alvo da Política Nacional de Atenção Integral à Saúde do Homem). A quantidade de participantes utilizada foi a sugerida pelo manual da TAP (Francis et al., 2004). Em sua maioria, os participantes eram casados (68\%), com filhos $(88 \%)$ e exerciam 
trabalho informal (72\%). Apesar de alfabetizados (76\%), muitos não acessaram o ensino formal (40\%) e apresentavam primeiro grau incompleto ( $80 \%)$.

\section{Instrumentos}

O instrumento utilizado foi um roteiro de entrevista semiestruturada, constituído por duas partes. A primeira parte apresentava: a) questões sociodemográficas, b) questões relacionadas à frequência com que os participantes comparecem à UBS e se consideram tal comportamento importante. A segunda parte contava com 7 perguntas abertas, com foco na avaliação do posto de saúde e na intenção de procurá-lo. As perguntas envolveram: c) levantamento das vantagens e desvantagens em procurar o posto de saúde, para identificação das crenças comportamentais salientes, d) levantamento das pessoas e/ou grupos influentes em procurar o posto de saúde, para identificação das crenças normativas salientes, e) levantamento das facilidades e dificuldades em procurar o posto de saúde, para identificação das crenças de controle salientes. As perguntas seguiram o modelo postulado pelos autores da Teoria da Ação Planejada (Ajzen, 1991). A tabela 1, a seguir, apresenta o roteiro da entrevista semiestruturada.

Tabela 1

Roteiro de perguntas da entrevista semiestruturada

$$
\text { Perguntas de caracterização da amostra }
$$

Conteúdo

Idade:

A

Estado civil:

A

Escolaridade:

A

B

B

Se sim, com que frequência?

( )uma vez ao ano ( )duas vezes ao ano

Você acha que os homens devem procurar a unidade básica de saúde? ( ) sim ( )não

Entre os homens que você convive, é comum procurar a unidade básica de saúde? ( ) sim ( )não

\section{Identificação das crenças}

1. Quais as vantagens de procurar a unidade básica de saúde anualmente por rotina?

2. Quais as desvantagens de procurar a unidade básica de saúde anualmente por rotina?

3. Que pessoas e/ou grupos lhe vêm à mente para procurar a unidade básica de saúde anualmente por rotina?

4. Que pessoas e/ou grupos aprovariam que você procurasse a unidade básica de saúde anualmente por rotina?

5. Que pessoas e/ou grupos desaprovariam que você procurasse a unidade básica de saúde anualmente por rotina?

\section{B}

B

C

C

D

$\mathrm{D}$

$\mathrm{D}$ 
6. Quais os fatores que facilitariam para que você procurasse a unidade básica de saúde anualmente por rotina?

7. Quais os fatores que dificultariam para que você procurasse a E unidade básica de saúde anualmente por rotina?

Nota. a) questões sociodemográficas, b) frequência com que os participantes comparecem à UBS e se consideram tal comportamento importante, c) identificação das crenças comportamentais salientes, d) identificação das crenças normativas salientes, e) identificação das crenças de controle salientes.

\section{Procedimentos}

A aproximação aos participantes da pesquisa ocorreu por intermédio dos agentes comunitários de saúde do distrito sanitário, que acompanharam as idas até a comunidade e domicílios, a fim de encontrar os homens cadastrados que corresponderiam aos critérios do estudo. Os critérios de exclusão foram: recusarse a participar da pesquisa, não estar em condições para responder as perguntas, não estar na faixa etária dos 25 a 59 anos e não estar cadastrado em alguma UBS do distrito sanitário de Maceió em questão.

Antes da coleta de dados, os participantes foram informados quanto aos objetivos deste estudo, bem como os riscos e benefícios envolvidos. Após a concordância, assinaram um termo de consentimento livre e esclarecido, indicando a autorização e o entendimento de todos os aspectos de investigação da pesquisa, conforme a Resolução No 466/12 sobre a pesquisa envolvendo seres humanos do Conselho Nacional de Saúde - CNS (Ministério da Saúde, 2012). O projeto foi aprovado pelo Comitê de Ética em Pesquisa (CEP) da Universidade Federal de Alagoas (UFAL) (parecer CAAE 43079214.8.0000.5013).

A entrevista foi realizada individualmente em domicílios, espaços comunitários e nas UBS's para maior comodidade dos participantes, sendo utilizado o gravador para o registro das falas. O tempo médio de entrevista foi de 9 minutos e 30 segundos. Foram levantadas as Crenças Comportamentais, Normativas e de Controle dos participantes, com o objetivo de se obter uma lista de "crenças modais salientes", ou seja, aquelas referentes ao comportamento pesquisado.

\section{Análise dos dados}

Os discursos foram organizados e analisados através da análise de conteúdo de Bardin (2011), sendo guiados pelas seguintes etapas: (a) transcrição de todas as crenças e referentes emitidos pela amostra, (b) leitura flutuante, (c) recorte das Unidades de Contexto Elementar (UCEs), (c) agrupamento das UCEs de significado semelhante, (d) organização das UCEs de significado semelhante em categorias, subcategorias e unidades temáticas (contribuição de três juízes - um doutor e dois alunos de pós-graduação), e (e) contagem da frequência por subcategoria, adotando o critério da frequência mínima, cujo valor deve ser igual ou superior a $75 \%$, com que cada Crença 
Comportamental, de Controle e Normativa foi eliciada para o comportamento em questão. A fim de facilitar a compreensão dos resultados, os trechos da entrevista a serem apresentados serão acompanhados da letra "P" (abreviação da palavra "participante"), seguido do número correspondente a ordem de realização da entrevista. Exemplo: P1.

\section{RESULTADOS}

A Tabela 2 apresenta dados descritivos em relação à procura pela UBS. Quando questionados se já haviam procurado a Unidade Básica de Saúde, a maioria dos participantes relatou já ter ido ao serviço $(80 \%)$ e frequentá-lo anualmente $(60 \%)$. Com relação à necessidade do homem ir à UBS, todos concordaram que os homens deveriam buscar o serviço. No entanto, quando perguntados se era comum aos homens do seu convívio procurarem a UBS, mais da metade (68\%) afirmou que esta não é uma prática rotineira.

Tabela 2

Conhecimentos relativos ao comportamento "Procurar a Unidade Básica de Saúde".

\begin{tabular}{ccc}
\hline Conhecimentos & Frequência & $\%$ \\
\hline Índice de procura à UBS & 20 & 80 \\
Sim & 5 & 20 \\
Não & & \\
Urequência da procura à & 15 & 60 \\
Anualmente & 5 & 20 \\
Mais de uma vez ao ano & 5 & 20 \\
Nunca & & \\
Concordância com a & 25 & 100 \\
procura masculina & 0 & 0 \\
Sim & & \\
Não & 8 & 32 \\
Procura à UBS pelos & 17 & 68 \\
homens do convívio & & \\
Sim & Não &
\end{tabular}

Em se tratando da análise das crenças salientes referentes à intenção masculina de procurar a UBS, de acordo com os construtos Atitude, Norma Subjetiva e Percepção de Controle, foram elencadas as categorias "Crenças Comportamentais", "Crenças Normativas" e "Crenças de Controle".

$\mathrm{Na}$ categoria "Crenças Comportamentais" foram obtidas 47 crenças salientes de um total de 52 crenças emitidas. De acordo com Moutinho e Roazzi 
(2010), de uma vasta exposição de crenças que as pessoas apresentam, apenas uma quantidade restrita se destaca como base da informação requisitada. Neste caso, buscavam-se as vantagens e desvantagens em procurar o posto de saúde. Essas crenças foram organizadas em oito dimensões. Percebeu-se uma proporção entre vantagens e desvantagens quanto à prática de procurar o posto de saúde. Dessa forma, quatro dimensões são de crenças positivas $(1,3,5,6)$ e as outras quatro são de crenças negativas $(2,4,7,8)$. A Tabela 3 apresenta frequência e porcentagem das crenças salientes (quantidade de participantes que manifestou cada crença) e as oito dimensões encontradas a partir delas.

Tabela 3

Crenças comportamentais modais salientes ao comportamento "Procurar a Unidade Básica de saúde"

\begin{tabular}{ccc}
\hline $\begin{array}{c}\text { Crenças comportamentais } \\
\text { modais salientes }\end{array}$ & Frequência & $\%$ \\
\hline 1 - Cuidar da saúde & 4 & 8,51 \\
2 - Não ter atendimento & 7 & 14,90 \\
3 - Acolhimento dos & 9 & 19,15 \\
profissionais & & 12,76 \\
$\begin{array}{c}\text { atendimento com } \\
\text { atraso }\end{array}$ & 6 & 14,90 \\
5 - Tratamento de doenças & 7 & 8,51 \\
6 - Atendimento próximo & 4 & 12,76 \\
$\quad$ de casa & 6 & 8,51 \\
7 - Faltar medicação & 4 & 100 \\
T- Não vê vantagem & & \\
Total de crenças modais & 47 &
\end{tabular}

Com relação à subcategoria "Cuidar da Saúde", a partir dos fragmentos de fala dos participantes, observa-se que estes consideram a UBS um local que possibilita não só aos homens, como à população em geral, uma maneira de estar cuidando da saúde, representando uma crença positiva: "É bom pra cuidar da saúde." (P15). "[...] É importante [...] Pra cuidar da saúde." (P18).

Já na subcategoria "Não ter atendimento", os participantes demonstraram, a partir de seus relatos, que as experiências anteriores de procurar a Unidade Básica de Saúde não foram benéficas, sendo caracterizadas pela negligência no atendimento por parte das equipes de saúde, atribuindo ao sistema de marcação de consulta o principal motivador desta crença negativa: "O ruim é porque às vezes você chega lá e não pode ser atendido, aí tem que agendar, pegar ficha." (P16). "O ruim é porque nem sempre você é atendido." (P17).

$\mathrm{Na}$ subcategoria "Acolhimento dos profissionais" foi encontrada a satisfação dos homens com a relação estabelecida com os profissionais que 
trabalham nas UBS's, sendo analisado não só o exercício da atividade profissional, mas também o acolhimento prestado: "O atendimento é bom, as pessoas são humanas..." (P4). "Bom atendimento [...] gente boa [...]" (P23).

$\mathrm{Na}$ subcategoria "Atendimento com atraso" falas descontentes com 0 tempo de espera para ser atendido nas UBS's foram apresentadas, em que muitos afirmaram não ter o desejo de buscar mais este serviço: "O ruim é porque às vezes você chega lá e não pode ser atendido [...] aí tem que agendar [...] pegar ficha [...] fica difícil." (P16). "O lado ruim é que numa precisão, se não tiver ficha, você não é atendido..." (P19).

Quanto à subcategoria "Tratamento de doenças", percebeu-se uma visão masculina da UBS enquanto serviço de saúde destinado exclusivamente ao tratamento de agravos, destacando a sua importância apenas para a melhoria das enfermidades: "O posto é uma casa que a gente precisa, uma emergência eu vou $[. .$.$] " (P11). "Só vou quando necessito [...] quando o estômago começa a$ doer." (P14).

No que concerne ao "Atendimento próximo de casa" os participantes referiram que as UBS's são serviços de saúde que oportunizam à população atendimento e tratamento das doenças perto de casa, demonstrando a representação positiva que fazem destes serviços: "Tem uma vantagem porque dá pra ir à pé, pra mim é bom porque faço uma caminhada." (P1). "A coisa boa de ir ao posto é porque é perto de casa." (P17).

A subcategoria "Faltar medicação" apresentou a relação que os homens fazem das UBS's com a precarização e falta de insumos presentes no serviço público, sendo considerado empecilho para que possam novamente buscar estes espaços: "A parte ruim é quando não tem remédio." (P23). "Precisa melhorar o medicamento [...] Passa uma semana e não tem medicamento [...] Às vezes a gente tem que comprar." (P24). Por fim, na subcategoria "Não vê vantagem", emitiu-se a ideia de que as UBS's não apresentam benefícios em suas vidas, podendo estar atrelado ao estilo de vida adotado pelos participantes.

Nas "Crenças normativas" com relação ao comportamento "Procurar a Unidade Básica de Saúde" foi obtido um total de 26 crenças normativas modais salientes, de um total de 32 crenças emitidas. Essas crenças foram subcategorizadas em cinco dimensões, conforme tabela 4.

Tabela 4

Crenças normativas modais salientes ao comportamento "Procurar a Unidade Básica de Saúde"

\begin{tabular}{ccc}
\hline $\begin{array}{c}\text { Crenças normativas modais } \\
\text { salientes }\end{array}$ & Frequência & $\%$ \\
\hline 1 - Esposa & 13 & 50 \\
2 - Família & 3 & 11,54 \\
3 - Filhos & 3 & 11,54 \\
\hline
\end{tabular}




$\begin{array}{ccc}4-\text { Irmãs } & 4 & 15,38 \\ 5 \text { - Si mesmo } & 3 & 11,54 \\ \text { Total de crenças modais } & 26 & 100 \\ \text { salientes } & & \end{array}$

Com base nos relatos dos participantes, a "esposa" apareceu como quem mais os incentivavam a cuidarem de si e a procurarem as UBS's: "Minha esposa me incentiva, diz: vá ao posto se consultar..." (P14). "Só quem me incentiva é a minha mulher [...] Ela sempre me incentiva a me cuidar." (P15). As "irmãs" e "mulheres da família" aparecem logo em seguida, como grandes apoiadoras do comportamento: "Minhas irmãs me incentivam..." (P3). "Muitas pessoas... várias... principalmente minha mãe e minha irmã." (P6). "Minha tia sempre me incentiva." (P19).

Menos frequente, mas também saliente, apareceu o referente "Si mesmo", representando a crença negativa de que para alguns homens a adesão a comportamentos de saúde depende única e exclusivamente da vontade deles, conforme as falas a seguir: "Ninguém especial... eu sou uma pessoa que eu mesma corro atrás." (P8). "Ninguém me incentiva. Sou eu mesmo que vou. Sempre fui. Desde garotão, 16, 17 anos. Sempre procurei nunca ninguém falou nada pra mim não." (P2). Os "vizinhos" também foram citados como rede social de suporte: "Meus vizinhos sempre falam." (P9). "Diariamente a vizinhança fala pra eu ir." (P11).

Por fim, no que se trata das "Crenças de controle modais salientes", foi obtido um total de 25 crenças de controle modais salientes, de um total de 39 crenças emitidas. Dessas crenças, surgiram 4 subcategorias, todas negativas, como apresentadas a seguir na tabela 5 .

Tabela 5

Crenças de controle modais salientes ao comportamento "Procurar a Unidade Básica de Saúde"

\begin{tabular}{ccc}
\hline $\begin{array}{c}\text { Crenças de controle modais } \\
\text { salientes }\end{array}$ & Frequência & $\%$ \\
\hline $\begin{array}{c}\text { obrigação } \\
\text { - Tomar medicaão por }\end{array}$ & 4 & 16 \\
$2 \begin{array}{c}\text { Não ter vaga para } \\
\text { atendimento }\end{array}$ & 4 & 16 \\
$3-$ Horário de trabalho & 8 & 32 \\
$4-$ Estar doente & 9 & 36 \\
Total de crenças modais & 25 & 100 \\
salientes & &
\end{tabular}

Na subcategoria "Tomar medicação por obrigação" percebeu-se que para grande parte dos homens entrevistados a procura pela UBS está relacionada à 
necessidade de tomar alguma medicação, sendo relatado que quando não há necessidade não existem motivos para procurar o serviço: "Eu vou para o posto porque tenho obrigação [...] Por causa da medicação... Se dissesse que não precisaria mais eu não iria... Só vou por causa da medicação." (P1). "O que me faz ir ao posto é porque é necessário ir, sou dependente do remédio." (P2).

$\mathrm{Na}$ subcategoria "Não ter vaga para atendimento", os participantes demonstraram, a partir de seus relatos, que as experiências anteriores de procurar a UBS não foram benéficas, sendo caracterizadas pela negligência no atendimento por parte das equipes de saúde, atribuindo ao sistema de marcação de consulta o principal motivador desta crença negativa: "Já desanimei de ir um bocado de vez [...] O que atrapalha é dar viagem perdida. Difícil ser atendido." (P14). "Difícil é ter a consulta." (P24).

A subcategoria "Horário de trabalho" demonstrou uma queixa masculina de que o horário de atendimento das UBS's não condiz com a realidade de trabalho da maioria dos homens, funcionando em horário similar à jornada de trabalho, aparecendo como um obstáculo ao comportamento: "Não vou quando eu estou trabalhando. Fica difícil o horário do posto porque você tem que pedir ao patrão e a pessoa que ganha salário mínimo $[\ldots]$ " (P2). "Pra gente que trabalha é difícil [...] Horário de trabalho [...]" (P7).

Por fim, a subcategoria "Estar doente", reforça a ideia de que o motivo para se buscar a UBS se restringe à presença de alguma enfermidade, recorrendo a esse serviço apenas por questões curativas: "O que faz com que eu não vá é porque eu nunca adoeci." (P7). "Só vou se tiver doente." (P18).

\section{DISCUSSÃO}

O presente estudo exploratório teve como objetivo identificar as crenças positivas e negativas e definir "as crenças modais salientes" relativas ao comportamento alvo "procurar a Unidade Básica de Saúde", em uma parcela da amostra dos homens de um distrito sanitário de saúde de Maceió ( $A L)$. Contouse com a participação de 25 homens, entre 25 e 59 anos, que responderam a um roteiro com perguntas semiestruturadas. De modo geral, pôde-se observar que para os homens participantes deste estudo a Intenção de procurar a UBS está associada às Crenças Comportamentais, Normativas e de Controle que permeiam o seu universo cognitivo e que, portanto, dificultam a realização do comportamento, conforme vem sendo apontado pela literatura (Gomes et al., 2011; Schraiber et al., 2010).

Algumas das crenças comportamentais encontradas neste estudo se assemelham com resultados de outras pesquisas (Albano et al., 2010; Brito, Santos, \& Maciel, 2010). A procura pela UBS está condicionada ao "Tratamento de doenças", sendo esta apontada como principal razão pela busca dos homens a estes serviços, ignorando as consultas preventivas (Brito, Santos, \& Maciel, 
2010). Além disso, identificou-se, também, "Atendimento com atraso" como fator que distancia o público masculino, caracterizada pelas intensas filas (Brito, Santos, \& Maciel, 2010). A crença masculina de que nas UBS's existe um bom acolhimento profissional, atribuindo ao cuidado prestado pelas equipes qualidades como "atencioso" e "respeitoso", assemelha-se à pesquisa de Gomes et al. (2011). Já a crença "Cuidar da Saúde", encontrada neste estudo, diferencia-se dos achados em pesquisas já realizadas (Alves et al., 2011; Gomes et al., 2011), onde o cuidado com a saúde aparece como uma expressão da necessidade feminina. As mulheres são vistas como seres mais frágeis, mais vulneráveis às doenças, necessitando de uma maior atenção à saúde.

A crença "Não ter vantagem" também se assemelha aos resultados encontrados por Storino et al. (2013), que destacam os discursos de homens que acreditam que a UBS não é capaz de atender às suas demandas, ou que não necessitam dos cuidados que lá são ofertados. Já a crença "Atendimento próximo de casa" assemelhou-se aos achados de Santos (2010), em que a distância das UBS's não se apresenta como impeditivo a acessibilidade masculina.

Quanto à crença "Faltar medicação", Couto et al. (2010) apresentam uma expressiva frequência masculina nas UBS's quando observada a busca por medicação nas farmácias, podendo associar isso à crença negativa de que a falta de medicação implica a não procura a estes locais.

Com base nos relatos dos participantes, a "esposa" apareceu como a referência modal saliente que mais os incentivaram a cuidarem de si e a procurarem as Unidades Básicas de Saúde, ratificando os dados da pesquisa de Schraiber et al. (2010), que concluíram que os homens casados procuram mais os serviços primários de saúde quando comparados aos homens solteiros.

As "irmãs" aparecem logo em seguida, como grandes apoiadoras do comportamento. Ambas, esposa e irmãs, levam à suposição de que as mulheres são mais influentes a esse comportamento do que os homens. Confirmando-se, portanto, a perspectiva da construção social da identidade masculina, que defende um modelo de invulnerabilidade aos homens, restando às mulheres o papel de olhar para o corpo e cuidar da saúde (Azevedo, 2007). Menos frequente apareceram vizinhos, filhos e família, mas estes também confirmam a importância da rede social de suporte no apoio à procura da UBS, dando destaque a família, como esteio essencial de compreensão e amparo (Nascimento \& Lemes, 2014).

Identificou-se, também, a representação do próprio participante como influência a realização do comportamento, confirmando a ideia de que quando o indivíduo tem a crença comportamental positiva para o comportamento, a sua atitude pode ser decisiva para a realização, assim como encontrado na pesquisa de Mendonça e Andrade (2010).

Corroborando com as crenças de controle salientes, investigações como a de Pinheiro et al. (2002) confirmam a crença negativa de "Não ter atendimento", 
sendo encontrados como motivadores a dificuldade de conseguir "vaga" ou "senha", a ausência do médico, a escassez de recursos e a longa espera. Aliado a isso, Azevedo (2007) pontua que a não valorização da demanda espontânea por parte dos profissionais que compõem as UBS's reverbera na negligência no atendimento dos homens que ali chegam. Indo mais além, Couto et al. (2010) trazem a lógica organizacional destes serviços como barreira ao atendimento do público masculino, em virtude do direcionamento das ações quase que exclusivamente às mulheres, crianças, idosos, hipertensos e diabéticos.

A crença "Horário de trabalho" é encontrada unanimemente nas pesquisas ligadas nessa área, que mostram o horário de funcionamento das UBS's como incompatíveis à jornada de trabalho na qual os homens estão submetidos, o que de certa forma desestimula com que estes acessem aqueles espaços (Alves et al., 2011; Santos, 2010). O Ministério da Saúde no processo de construção PNAISH (Ministério da Saúde, 2009) declarou que existe de fato uma preocupação masculina com a atividade laboral, sobretudo em pessoas de baixa condição social, o que reforça o papel historicamente atribuído ao homem de ser responsável pelo sustento da família. Porém, destacou que apesar de essa ser uma barreira importante, atualmente grande parte das mulheres, de todas as categorias socioeconômicas, estão inseridas no mercado de trabalho formal e nem por isso deixam de procurar os serviços de saúde.

As crenças "Tomar medicação por obrigação" e "Estar doente" reforçam os resultados de trabalhos como o Pinheiro et al. (2002), que demonstram que os homens quando procuram serviços de saúde, como as UBS's, o fazem por questões ligadas à doença, problema odontológico, tratamento ou reabilitação e acidente ou lesão.

\section{CONSIDERAÇÕES FINAIS}

A partir das crenças encontradas neste estudo, pode-se considerar que o comportamento de procurar a Unidade Básica de Saúde por parte da população masculina está diretamente relacionado às crenças comportamentais, normativas e de controle que permeiam o universo cognitivo desses homens. Observou-se que as crenças comportamentais podem tanto favorecer quanto dificultar a intenção de realizar o comportamento. Associado a isso, as crenças normativas demonstram que o fato da rede social, que normalmente incentiva 0 comportamento masculino, não envolver os pares de mesmo gênero parece ter repercutido negativamente para a sua efetivação. Por fim, as crenças de controle, mesmo apresentando dimensões que, em tese, favorecem a busca masculina pelos serviços das Unidades Básicas de Saúde, quando analisadas, configuram representações negativas do comportamento, como no caso da crença de tomar uma medicação por obrigação. 
Dessa forma, os conteúdos aqui encontrados podem servir de indicadores para futuras campanhas estimuladoras ao cuidado à saúde masculina, assim como já vem sendo utilizadas com as mulheres, nos casos de prevenção ao câncer de mama e ao câncer de colo do útero. Aliado a isso, servem de parâmetros para a criação de estratégias que favoreçam o acesso dos homens às UBS's, reorganizando estes serviços e qualificando suas equipes. Considera-se que a prevenção em saúde a partir dos serviços primários evita a ocorrência de muitos agravos, além de ser menos oneroso aos cofres públicos.

Estudos com amostras mais amplas sobre os efeitos das comunicações persuasivas na modificação dessas crenças parecem bastante promissores, visando-se um melhor entendimento da ausência masculina nas UBS's, consequentemente, um maior índice de procura à UBS e um menor grau de absenteísmo em nosso país. Dados relativos a formas mais eficazes de intervenção a partir das mídias têm um potencial elevado de esclarecimento e influência de comportamentos ajustados.

Por fim, indicamos que pesquisas futuras busquem diversificar sua amostra pois, mesmo considerando as contribuições deste trabalho, foram utilizados na amostra somente homens que eram cadastrados em UBS's de um distrito sanitário de Maceió. A especificidade do ambiente social da amostra limita a variação de opiniões. Podendo considerar, também, homens que não estejam cadastrados em UBS's.

\section{DECLARAÇÃO DE CONFLITOS DE INTERESSE}

As autoras declaram que não houve conflitos de interesse.

\section{REFERÊNCIAS}

Ajzen, I. (1991). The theory of planned behavior. Organizational Behavior and Human Decision Process, 50(2), 179-211. doi:10.1016/07495978(91)90020-T

Ajzen, I., \& Fishbein, M. (1980). Understanding attitudes and predicting social behavior. Englewood Cliffs: Prentice-Hall.

Albano, B. R., Basílio, M. C., \& Neves, J. B. (2010). Desafios para a inclusão dos homens nos serviços de atenção primária à saúde. Revista Enfermagem Integrada, 3(2), 554-563. Recuperado de https://www.unilestemg.br/enfermagemintegrada/artigo/V3 2/08-desafiospara-inclusao-dos-homens-em-servicos-primarios-de-saude.pdf.

Alves, R. F., Silva, R. P., Ernesto, M. V., Lima, A. G.B., \& Souza, F. M. (2011). Gênero e saúde: O cuidar do homem em debate. Psicologia: Teoria e Prática, 13(3), 152-166. Recuperado de http://pepsic.bvsalud.org/pdf/ptp/v13n3/v13n3a12.pdf. 
Armitage, C. J. (2005). Can the theory of planned behavior predict the maintenance of physical activity?. Health Psychology, 24(3), 235. doi: 10.1037/0278-6133.24.3.235.

Azevedo, A. M. A. (2007). Acesso à atenção à saúde no SUS: o PSF como (estreita) porta de entrada. (Dissertação de Mestrado). Instituto Aggeu Magalhães, Recife.

Bandura, A. (1986). Social foundations of thought and action. Englewood Cliffs: Prentice-Hall.Crawford.

Bardin, L. (2011). Análise de conteúdo. São Paulo, SP: Edições 70.

Brito, R. S., Santos, D. L. A., \& Maciel, P. S. O. (2010). Olhar masculino acerca do atendimento na Estratégia Saúde da Família. Revista Rene, 41(4), 135142. http://dx.doi.org/10.15253/rev\%20rene.v11i4.4635.

Bryan, A., Fisher, J. D., \& Fisher, W. A. (2002). Tests of the mediational role of preparatory safer sexual behavior in the context of the theory of planned behavior. Health Psychology, 21(1), 71. http://psycnet.apa.org/doi/10.1037/0278-6133.21.1.71.

Conner, M., Norman, P., \& Bell R. (2002). The theory of planned behavior and healthy eating. Health Psychology. 21(2), 194-201. http://psycnet.apa.org/doi/10.1037/0278-6133.21.2.194.

Conner, M., Warren, R., Close, S., \& Sparks, P. (1999). Alcohol Consumption and the Theory of Planned Behavior: An examination of the cognitive mediation of past behavior id. Journal of Applied Social Psychology, 29(8), 1676-1704. https://doi.org/10.1111/j.1559-1816.1999.tb02046.x.

Courneya, K. S. (1995). Understanding readiness for regular physical activity in older individuals: an application of the theory of planned behavior. Health Psychology, 14(1), 80-87. http://psycnet.apa.org/doi/10.1037/02786133.14.1.80.

Courneya, K. S., \& Friedenreich, C. M. (1999). Utility of the theory of planned behavior for understanding exercise during breast cancer treatment. Psycho-Oncology, 8(2), 112-122. doi:10.1002/(SICI)10991611(199903/04)8:2<112::AID-PON341>3.0.CO;2-L.

Courtenay, W. (2000). Constructions of masculinity and their influence on men's well-being: A theory of gender and health. Social Science \& Medicine, 50(10), 1385-401. https://doi.org/10.1016/S0277-9536(99)00390-1.

Couto, M. T., Pinheiro, T. F., Valença, O., Machin, R., Silva, G. S. N., Gomes, R., ..., \& Figueiredo, W. S. (2010). O homem na atenção primária à Saúde: Discutindo (in)visibilidade a partir da perspectiva de gênero. Interface Comunicação, Saúde, Educação, 14(33), 257-270. doi:10.1590/S141432832010000200003.

D'amorim, M. A. (2000). A teoria da ação racional nos comportamentos de saúde. Temas em Psicologia, 8(1), 39-44. Recuperado de http://pepsic.bvsalud.org/pdf/tp/v8n1/v8n1a05.pdf. 
D'amorim, M. A., Freitas, C. M., \& Sá, G. A. C. (1992). A motivação para realizar o auto-exame da mama: Uma aplicação da teoria da ação planejada. Psicologia: Teoria e Pesquisa, 8(2), 169-185.

Fishbein, M., \& Ajzen, I. (1975). Belief, attitude, intention, and behavior: An introduction to theory and research. Reading, MA: Addison-Wesley.

Francis, J. J., Eccles, M. P., Johnston, M., Walker, A., Grimshaw, J., Foy, R., ..., \& Bonetti, D. (2004). Constructing questionnaires based on the theory of planned behaviour: A manual for health services researchers. Newcastle upon Tyne, UK: Centre for Health Services Research, University of Newcastle upon Tyne. Recuperado de http://openaccess.city.ac.uk/id/eprint/1735.

Gomes, R., Moreira, M. C. N., Nascimento, E. F., Rebello, L. E. F. S., Couto, M. T., Schraiber, L. B. (2011). Os homens não vêm! Ausência e/ou invisibilidade masculina na atenção primária. Ciência \& Saúde Coletiva, 16 (11), 983-992. doi:10.1590/S1413-81232011000700030.

Gonçalves, S. C. M., \& Dias, M. R. (1999). A prática do autoexame da mama em mulheres de baixa renda: um estudo de crenças. Estudos de Psicologia, 4(1), 141-159. doi:10.1590/S1413-294X1999000100008.

Harakeh, Z., Scholte, R. H., Vermulst, A. A., Vries, H., \& Engels, R. C. (2004). Parental factors and adolescents' smoking behavior: An extension of the theory of planned behavior. Preventive Medicine, 39(5), 951-961. doi: 10.1016/j.ypmed.2004.03.036.

Instituto Brasileiro de Geografia e Estatística (2016). Tábua completa de mortalidade para o Brasil: Breve análise da evolução da mortalidade no Brasil. Rio de Janeiro, RJ: IBGE. Recuperado de ftp://ftp.ibge.gov.br/Tabuas Completas de Mortalidade/Tabuas Completas de Mortalidade 2015/tabua de mortalidade analise.pdf.

Maciel, M. G., \& Veiga, R. T. (2012). Intenção de mudança de comportamento em adolescentes para a prática de atividades físicas de lazer. Revista Brasileira de Educação Física e Esporte, 26(4), 705-16. doi:10.1590/S180755092012000400014.

Matos, E. B., Veiga, R. T., \& Reis, Z. S. N. (2009). Intenção de uso de preservativo masculino entre jovens estudantes de Belo Horizonte: Um alerta aos ginecologistas. Revista Brasileira de Ginecologia \& Obstetrícia, 31(11), 574-580. http://dx.doi.org/10.1590/S0100-72032009001100008.

Mendonça, V. S., \& Andrade, A. N. (2010). A Política Nacional de Saúde do Homem: Necessidade ou ilusão? Psicologia Política, 10(20), 216-226. Recuperado de http://pepsic.bvsalud.org/pdf/rpp/v10n20/v10n20a03.pdf.

Ministério da Saúde. (2009). Política Nacional de Atenção Integral à Saúde do Homem: Princípios e diretrizes. Brasília, DF: Ministério da Saúde. Recuperado de http://www.unfpa.org.br/Arquivos/saude do homem.pdf. 
Ministério da Saúde. (2012). Resolução n. 466. Brasília, DF: Diário Oficial da União.

http://bvsms.saude.gov.br/bvs/saudelegis/cns/2013/res0466 12122012. html.

Moura, E. C., Santos, W., Neves, A. C. M., Gomes, R., \& Schawrz, E. (2014). Atenção à Saúde dos homens no âmbito da Estratégia de Saúde da Família. Ciência \& Saúde Coletiva, 19(2), 429-438. doi:10.1590/141381232014192.05802013.

Moutinho, K., \& Roazzi, A. (2010). As teorias da ação racional e da ação planejada: relações entre intenções e comportamentos. Avaliação Psicológica, 9(2), 279-287. Recuperado de http://pepsic.bvsalud.org/pdf/avp/v9n2/v9n2a12.pdf.

Nascimento, V. F., \& Lemes, A. G. (2014). Saúde do homem: Sentimento de masculinidade comprometida. Revista Eletrônica Gestão e Saúde, 5(1), 8090. http://dx.doi.org/10.18673/gs.v5i1.22827.

Pinheiro, R. S., Viacava, F., Travassos, C., \& Brito, A. S. (2002). Gênero, morbidade, acesso e utilização de serviços de saúde no Brasil. Ciência \& Saúde Coletiva, 7(4), 687-7070. http://dx.doi.org/10.1590/S141381232002000400007.

Sabo, D. (2002). O estudo crítico das masculinidades. In. M. Adelman, \& C. B. Silvestrin (Eds.), Coletânea gênero plural. Curitiba, PR: Editora UFPR.

Santos, F. A. (2010). Dinâmica da acessibilidade masculina ao Programa de Saúde da Família. (Dissertação de Mestrado). Universidade Estadual do Sudoeste da Bahia, Vitória da Conquista.

Schraiber, L. B., Figueiredo, W. S., Gomes, R., Couto, M. T., Pinheiro, T. F., Machin, R., ..., \& Valença, O. (2010). Necessidades de saúde e masculinidades: Atenção primária no cuidado aos homens. Cadernos de Saúde Pública, 26(5), 961-970. http://dx.doi.org/10.1590/S0102311X2010000500018.

Storino, L. P., Souza, K. V., \& Silva, K. L. (2013). Necessidades da saúde de homens na atenção básica. Escola Anna Nery, 17(4), 638-645. doi: 10.5935/1414-8145.20130006. 
Sobre as autoras

Jéssica Bazilio Chaves é Terapeuta Ocupacional pela Universidade Estadual de Ciências da Saúde de Alagoas, mestre em Psicologia pela Universidade Federal de Alagoas. jessicabazilioc@gmail.com.

Sheyla Christine Santos Fernandes é psicóloga e mestre em Psicologia Social pela Universidade Federal da Paraíba, doutora em Psicologia Social pela Universidade Federal da Bahia. Atua como docente da graduação e da pós-graduação em Psicologia da Universidade Federal de Alagoas. sheylacsfernandes@msn.com. Daniela Santos Bezerra é psicóloga pela Universidade Federal de Alagoas. danielabezerra.psicologia@gmail.com.

J.B.C. e S.C.S.F. contribuíram para a conceitualização, investigação e visualização do artigo; J.B.C. fez a redação inicial do artigo (rascunho) e D.S.B. e S.C.S.F. são as responsáveis pela redação final (revisão e edição).

Recebido em: 26/10/2016

Revisado em: 20/03/2017

Aceito em: 18/09/2017 